\title{
PocketGraph: graph representation of binding site volumes
} M Weisel* , J Kriegl and G Schneider

Address: Johann Wolfgang Goethe-University, Beilstein Endowed Chair for Cheminformatics, Siesmayerstraße 70, D-60323 Frankfurt/Main, Germany

* Corresponding author

from 4th German Conference on Chemoinformatics

Goslar, Germany. 9-II November 2008

Published: 5 June 2009

Chemistry Central Journal 2009, 3(Suppl I):P66 doi:10.1186/1752-153X-3-SI-P66

This abstract is available from: http://www.journal.chemistrycentral.com/content/3/SI/P66

(C) 2009 Weisel et al; licensee BioMed Central Ltd.

The representation of small molecules as molecular graphs [1] is a common technique in various fields of cheminformatics. This approach employs abstract descriptions of topology and properties for rapid analyses and comparison. Receptor-based methods in contrast mostly depend on more complex representations impeding simplified analysis and limiting the possibilities of property assignment. In this study we demonstrate that ligand-based methods can be applied to receptor-derived binding site analysis.

We introduce the new method PocketGraph that translates representations of binding site volumes into linear graphs and enables the application of graph-based methods to the world of protein pockets. The method uses the PocketPicker [2] algorithm for characterization of binding site volumes and employs a Growing Neural Gas [3] procedure to derive graph representations of pocket topologies.

Self-organizing map (SOM) projections revealed a limited number of pocket topologies. We argue that there is only a small set of pocket shapes realized in the known ligandreceptor complexes.

\section{References}

I. Balaban AT: Applications of Graph Theory in Chemistry. J Chem Inf Comput Sci 1985, 25:334-343.

2. Weisel M, Proschak E, Schneider G: PocketPicker: Analysis of Ligand Binding-Sites with Shape Descriptors. Chem Cent J 2007, I:7.

3. Fritzke B: Growing cell structures - a selforganizing network for unsupervised and supervised learning. Neural Networks 1994, 7:144|-1460. 